\title{
In Situ Observation of Single Ion Damage in Electronic Materials
}

\author{
Daniel Bufford ${ }^{1}$, Remi Dingreville ${ }^{1}$ and Khalid Hattar ${ }^{1}$ \\ 1. Sandia National Laboratories, Albuquerque, NM, USA
}

Radiation damages crystalline materials by displacing atoms from their lattice sites, a process that may degrade material properties. In semiconductor components, device performance may deteriorate gradually with increasing fluence due to accumulated damage, however, sub-micrometer length scale device components are also susceptible to sudden problems. These "single event effects", so called because they are caused by a single particle [1], range from transient errors (e.g. flipping of a memory bit), to permanent losses of device functionality, as in gate rupture. With increasingly shorter length design rules, a single cascade may affect an entire element, or even span multiple elements. Even in metals, single ions are capable of creating dislocation structures, or even craters and cavities, all of which reduce electrical conductivity and overall performance.

Although some models exist to simulate this radiation-induced defect production in $\mathrm{Si}$, many do not extend past cascade quenching, i.e. time scales on the order of tens of picoseconds. Defect clusters in $\mathrm{Si}$ are known to evolve on much longer time scales, stretching to seconds or minutes [2]. In order to better inform computational models currently under development to simulate this behavior on longer time scales, this study observed and characterized defects produced in commonly used electronic materials by single ions as a function of ion atomic mass, energy, and sample temperature. Ion irradiation performed in situ inside of a TEM can provide real time observation of defects produced by irradiation, alongside the powerful suite of microstructural characterization tools the microscope offers. The resulting experimental data approach the time and length scales intended to be addressed by the model. To this end, $\mathrm{Si}$ and $\mathrm{Au}$ were irradiated during in situ observation, and the resulting defects were characterized and allowed to age for different times at a range of relevant temperatures.

In this work, gold samples were prepared by depositing films onto $\mathrm{NaCl}$ substrates by pulsed laser deposition, then floating the films off in water and collecting them on Mo mesh TEM grids. These samples were then annealed for 4 hours at $400{ }^{\circ} \mathrm{C}$ in a vacuum furnace $\left(\sim 10^{-7}\right.$ torr $)$ to coarsen the grains for improved imaging and analysis. Si samples were prepared from single crystal wafers by grinding, polishing, and low-angle ion milling. Additional Si samples were prepared by the small angle cleavage technique [3], which avoids the surface damage caused by ion milling. The in situ ion irradiation experiments were completed at the in situ ion irradiation TEM ( $\left.{ }^{3} \mathrm{TEM}\right)$ facility located at Sandia National Laboratories [4]. This facility includes a JEOL 2100 TEM connected to an EN Tandem Van de Graaff accelerator (0.8-6 MV), among other equipment. To reliably distinguish single ion strikes, the ion beam flux incident on the sample was at most $10^{9}$ ions $\mathrm{cm}^{2} \mathrm{~s}^{-1}$, which corresponds to approximately 1 ion s$~^{-1}$ within the $330 \mathrm{~nm} \times 330 \mathrm{~nm}$ viewing field at $\times 100,000$ magnification. Video was collected during these experiments to identify the locations of and damage resulting from single ion strikes (see Figure 1), and defects were characterized using bright- and dark-field imaging techniques.

Single ions in Si produce locally disordered regions in the vicinity of the cascade. With sufficient ion fluence, these regions overlap and eventually lead to a disordered layer. In contrast, Au remains crystalline during irradiation, with cascades generating an array of point defects, dislocation loops, and stacking fault tetrahedra (Figure 2). The behavior of these various types of radiation-induced defects in the two materials in this study will be compared to previous experimental work, and to various previously developed models. 


\section{References}

[1] JR Schwank, et al, IEEE Transactions on Nuclear Science 60 (2013), p.2074-2100.

[2] PD Edmondson, et al, Solid State Phenomena 108-109 (2005), p.145-150.

[3] JP Mccaffrey, Ultramicroscopy 38 (1991), p.149-157.

[4] K Hattar, et al, Nuclear Instruments \& Methods in Physics Research Section B-Beam Interactions with Materials and Atoms 338 (2014), p.56-65.

[6] The authors thank X. Zhang (Texas A\&M University), D.L. Buller and B.R. Muntifering for their assistance. Sandia National Laboratories is a multi-program laboratory managed and operated by Sandia Corporation, a wholly owned subsidiary of the Lockheed Martin Corporation, for the U.S. Department of Energy's National Nuclear Security Administration under contract DE-AC0494AL85000.
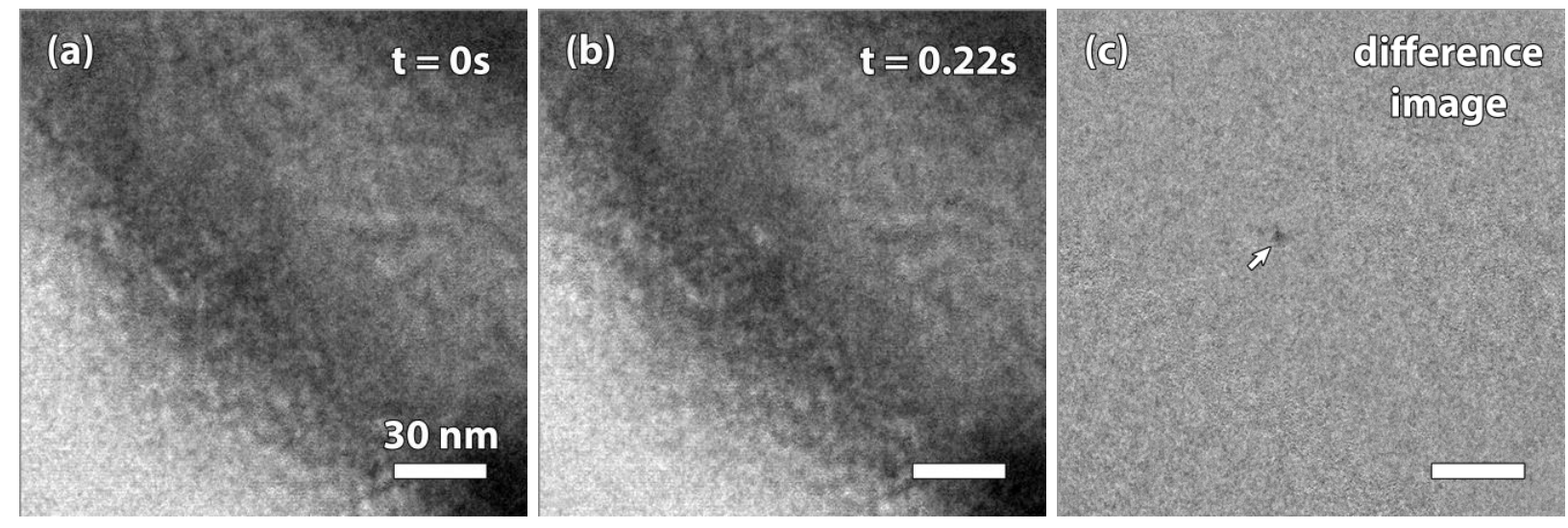

Figure 1. Sequential transmission electron micrograph snapshots from video collected in situ during ion irradiation of $\mathrm{Si}$ with $46 \mathrm{keV} \mathrm{Au}^{1-}$. (a,b) Two sequential frames capturing the creation of a defect cluster. (c) Difference image: here features present in (a) but not (b) appear light, and those in (b) but not (a) appear dark. Unchanged features make up the flat, lighter grey background. Hence, the defect created defect (arrow) is more easily seen.
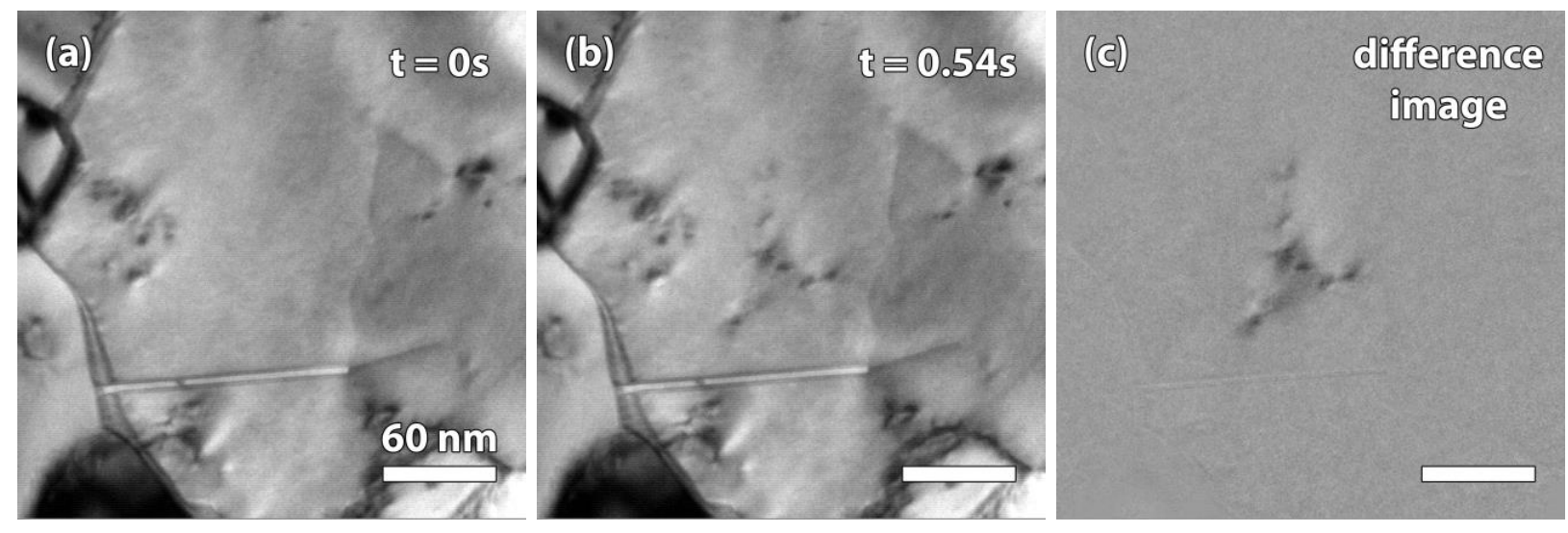

Figure 2. Sequential transmission electron micrograph snapshots from video collected in situ during ion irradiation of $\mathrm{Au}$ with $2.8 \mathrm{MeV} \mathrm{Au}^{4+}$. (a) Here a several defect clusters are visible from previous single Au ions. (b) Another cluster of defects appears in the center. (c) The difference image shows the extent of the damage caused by the ion in (b). 